\title{
Clinical Significance of Transient Left Ventricular Dilation Assessed during Myocardial Tc-99m Sestamibi Scintigraphy
}

\author{
Paulo Schiavom Duarte, Paola Emanuela Smanio, Carlos Alberto O liveira, Luiz Roberto Martins, \\ Luiz Eduardo Mastrocolla, Julio César Pereira
}

São Paulo, SP - Brazil

\begin{abstract}
Objective - To assess the clinical significance of transient ischemic dilation of the left ventricle during myocardial perfusion scintigraphy with stress/rest sestamibi.
\end{abstract}

Methods - The study retrospectively analyzed 378 patients who underwent myocardial perfusion scintigraphy with stress/rest sestamibi, 340 of whom had a low probability of having ischemia and 38 had significant transient defects. Transient ischemic dilation was automatically calculated using Autoquant software. Sensitivity, specificity, and the positive and negative predictive values were established for each value of transient ischemic dilation.

Results - The values of transient ischemic dilation for the groups of low probability and significant transient defects were, respectively, $1.01 \pm 0.13$ and $1.18 \pm 0.17$. The values of transient ischemic dilation for the group with significant transient defects were significantly greater than those obtained for the group with a low probability $(P<0.001)$. The greatest positive predictive values, around $50 \%$, were obtained for the values of transient ischemic dilation above 1.25 .

Conclusion - The results suggest that transient ischemic dilation assessed using the stress/rest sestamibi protocol may be useful to separate patients with extensive myocardial ischemia from those without ischemia.

Key words: transient left ventricular dilation, myocardial ischemia, sestamibi scintigraphy

Nuclear Medicine and Cardiology Unit, Fleury - Centro de Medicina Diagnóstica and Faculdade de Saúde Pública of USP

Mailing address: Paulo Schiavom Duarte - Seção de Medicina Nuclear

Rua Cincinato Braga, 282 - Cep 01333-910 - São Paulo, SP, Brazil

E-mail: paulo.duarte@fleury.com.br

Received 10/23/02

Accepted 1/14/03

English version by Stela Maris C. e Gandour
The balanced involvement of multiple arteries in coronary artery disease is a potential cause of false-negative results on myocardial perfusion scintigraphy. During exercise, that degree of coronary artery disease may cause perfusion defects homogeneously distributed throughout the myocardium, leading to uniform radiopharmaceutical upta$\mathrm{ke}$, and, consequently, to a false-negative examination ${ }^{1}$.

Transient dilation of the left ventricle - also known as transient ischemic dilation (TID) - is considered present when the image of the left ventricular cavity seems to be significantly greater after stress as compared with that at rest. Transient ischemic dilation is a way to detect balanced coronary artery disease in patients with apparently normal myocardial perfusion scintigraphy.

Transient ischemic dilation has been classically described in myocardial perfusion examinations performed with the stress/redistribution thallium-201 protocol $^{1,2}$. In this protocol, the images following stress are obtained almost immediately after radiopharmaceutical injection, during the period when the myocardium is under the effects of physical stress. However, this signal has also been described in other myocardial perfusion scintigraphy protocols, such as the stress/rest sestamibi protocol ${ }^{3}$, the tetrofosmin stress/rest protocol ${ }^{4}$, or the dual-isotope protocol (thallium rest/sestamibi stress) $)^{5}$. In these situations, the acquisition of images representative of perfusion under stress is performed some time after the exercise test (30 to 60 minutes), when the myocardium has already had time to recover from the mechanical dysfunction caused by stress. Transient ischemic dilation has also been described in situations in which the myocardium has not undergone a real stress situation, such as in the examination performed with the pharmaceutical stimulus of dipyridamole or adenosine ${ }^{6-9}$. Thus, some researchers consider that the term dilation is imprecise and should represent, in most cases, an apparent dilation secondary to diffuse subendocardial ischemia. Although transient ischemic dilation has been frequently reported in the literature, scarce information exists about the real value of that parameter to assess coronary artery disease and about the values to be used to better separate the patients with exten- 
sive myocardial ischemia from those without ischemia, mainly, when the myocardial perfusion study is performed according to the 1-day protocol with stress/rest sestamibi.

\section{Methods}

This study aimed at assessing whether transient ischemic dilation may be a useful index when observed in myocardial perfusion scintigraphy performed according to the 1-day protocol with stress/rest sestamibi, and at trying to establish values of transient ischemic dilation to separate patients with extensive myocardial ischemia from those with a low probability of having myocardial ischemia.

Three hundred and seventy-eight patients were retrospectively studied. These patients had been referred to the nuclear medicine unit to undergo myocardial perfusion scintigraphy according to the 1-day protocol with stress/ rest sestamibi. Of the 378 patients, 340 (mean age, 52 years; 259 men, 81 women) had a low probability of ischemia (sum of the stress scores $-\mathrm{SSS}<2$, negative exercise test, no previous history of infarction, myocardial revascularization, angioplasty, or typical angina) and 38 (mean age, 59 years; 32 men, 6 women) had extensive transient defects (sum of the differences of the scores - SDS >9).

It is worth noting that the referred perfusion scores are analyses of perfusion in different myocardial segments and may be established through subjective visual assessment or automatically calculated with processing programs. In our study, the scores were automatically established with Autoquant software and adjusted by the nuclear physician, when necessary. The scores, as well as transient ischemic dilation, are discussed in the I Guideline on Nuclear Cardiology published by the Brazilian Society of Cardiology ${ }^{10}$.

The 1-day protocol with sestamibi in the rest and stress phases was used for acquisition of tomographic images (SPECT) of myocardial perfusion. The images at rest were acquired 30 minutes after injection of $370 \mathrm{MBq}(10 \mathrm{mCi})$ of Tc-99m sestamibi. The stress phase was performed 4 hours after the rest phase. Cardiac stress was induced by programmed exercise on a treadmill according to one of the following protocols: Bruce, modified Bruce, or Ellestad protocol. During exercise peak or when the patient had limiting symptoms, 1.11 $\mathrm{GBq}(30 \mathrm{mCi})$ of sestamibi was intravenously injected. The images were acquired 45 to 60 minutes after injection. A scintillation camera with 2 detectors (Vertex plus MCD-AC) equipped with low energy and high resolution collimator was used. Forty-eight projections ( $25 \mathrm{~s} /$ projection) were obtained using a 64 x 64 matrix and a sweeping angle of $180^{\circ}$.

Data were processed with the Ultra-60 workstation (SUN Microsystems, Santa Clara, CA, USA). The reconstruction was performed according to the technique of filtered retro-projection and Butterworth filter using Autospect plus software (Philips Medical Systems, Andover, MA, USA). Correction was made with the movement, when necessary. Transient ischemic dilation was automatically calculated using Autoquant software (Philips Medical Systems, Andover, MA, USA), which calculates the value of transient ischemic dilation by dividing the volume of the left ven- tricular cavity after effort by the volume of the left ventricular cavity at rest (volume of the left ventricular cavity during stress/volume of the left ventricular cavity at rest).

The mean and standard deviation (SD) for that index were calculated for the 2 groups of patients. The nonpaired $t$ test was used to compare the means of the values of transient ischemic dilation for the groups. Sensitivity, specificity, and the positive and negative predictive values were established for each value of transient ischemic dilation, ranging from 0.7 to 1.6 to separate the group of patients with reversible defects from those with a low probability of having myocardial ischemia. The ROC curve was also plotted.

\section{Results}

The transient ischemic dilation values for the groups with a low probability and significant transient defects were $1.01 \pm 0.13$ and $1.18 \pm 0.17$ (mean $\pm S D$ ), respectively. The distribution of the values of transient ischemic dilation for the 2 groups of patients is shown in figure 1 . The mean values of transient ischemic dilation for the group with significant transient defects was significantly greater than those obtained for the group with a low probability $(\mathrm{P}<0.001)$ (fig. 2$)$. Sensitivity, specificity, and the positive and negative predictive values for the different transient ischemic dilation values are shown in figure 3. The ROC curve (fig. 4) showed an area below the curve of 0.79 , with a $95 \%$ confidence interval ranging from 0.703 to 0.876 , and a better relation between sensitivity and specificity (the most distant point from the diagonal line) at the transient ischemic dilation value of 1.05. At this point, the test had a sensitivity of $82 \%$ and a specificity of $69 \%$.

\section{Discussion}

Transient ischemic dilation is a parameter obtained on

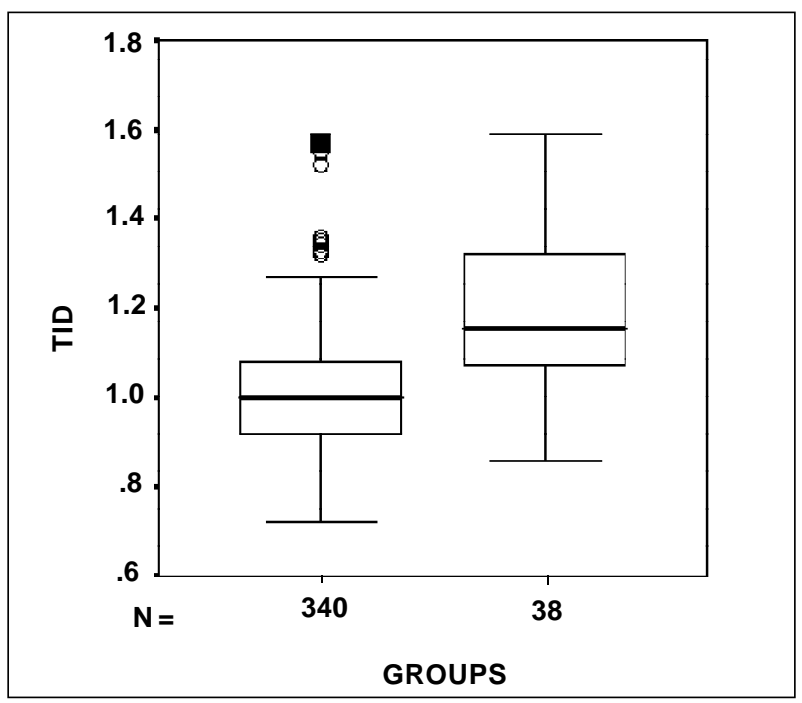

Fig. 1 - Box plot of the distribution of the transient ischemic dilation values for the 2 groups of patients. The transversal bar inside the box shows the median of the values. The box groups $50 \%$ of the values closer to the median, and the vertical bar groups the maximum and minimum values (I) except for the outlier $(\mathrm{O})$ and extreme (⿷) values. 
myocardial perfusion examination and may be automatically calculated with some types of software. This parameter may be useful to detect extensive and balanced coronary artery disease in patients with normal myocardial perfusion ${ }^{1}$. It was classically described using the protocol with stress/redistribution thallium ${ }^{1,2}$, but may be present when other protocols, such as the stress/rest sestamibi ${ }^{3}$ or the dual-isotope protocols, are used ${ }^{5}$. However, the transient ischemic dilation value in the 2 latter situations has been less analyzed.

This study analyzed whether the transient ischemic dilation value automatically calculated with Autoquant software is associated with moderate to extensive myocardial ischemia detected on the myocardial perfusion examinations performed using the 1-day protocol with rest/stress sestamibi. Because the cardiac catheterization results of patients were not available, a group of patients with balanced triplevessel coronary disease could not be defined. For the purpose of this study, the patients with moderate to extensive reversible defects on myocardial perfusion examination were considered to have a transient ischemic dilation value similar to that of patients with balanced triple-vessel coronary disease.

The results obtained showed a significant difference between the mean values of transient ischemic dilation in the group of patients with reversible defects and in the group of patients with a low probability of having ischemia. Figure 3 shows that, although sensitivity and specificity reached values close to $75 \%$ for transient ischemic dilation values around 1.07 , the positive predictive value was very low for this degree of transient ischemic dilation. The positive predictive value progressively increased for transient ischemic dilation values ranging from around 0.90 to 1.25 , where positive predictive values close to $50 \%$ were obtained; beyond that point, the positive predictive value began to fluctuate. This fluctuation was due to the presence of a few patients with transient ischemic dilation values greater

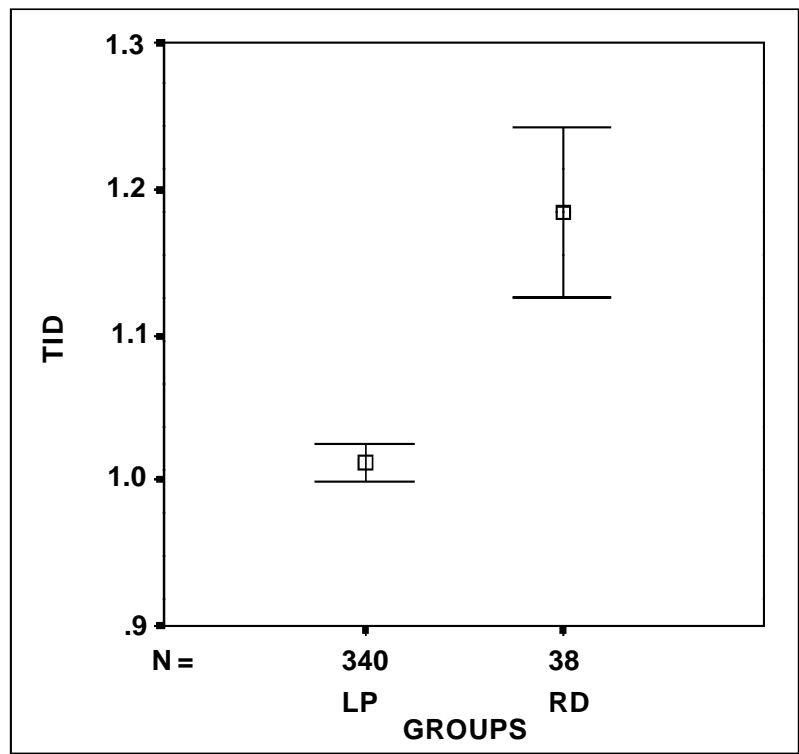

Fig. 2 - Error bar of the means ( $\square$ ) for the 2 groups of patients with a $95 \%$ confidence interval (I).

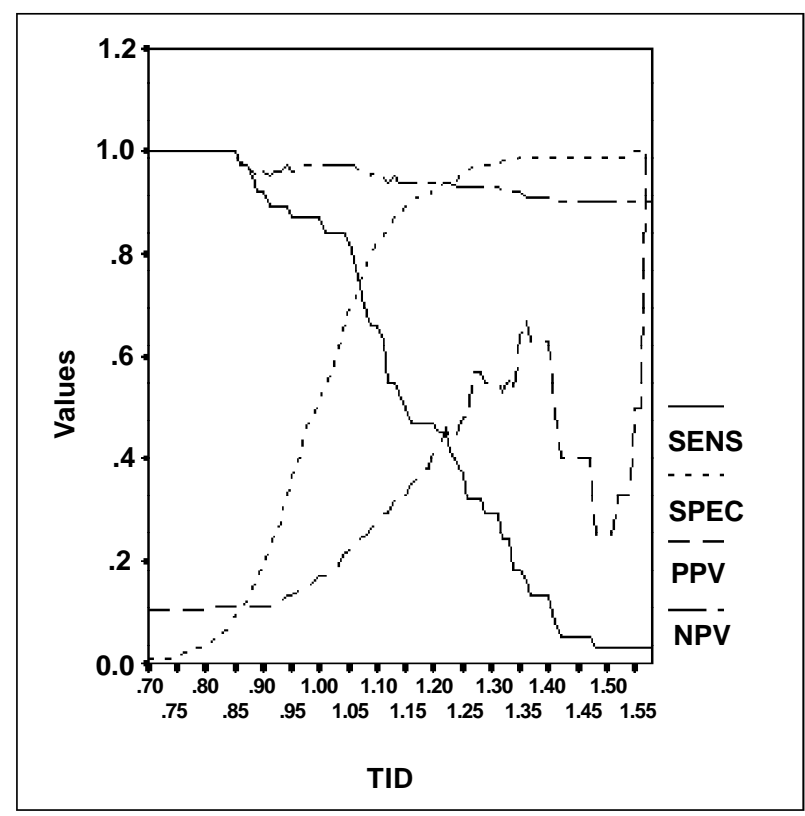

Fig. 3 - Sensitivity (SENS), specificity (SP), positive predictive value (PPV), and negative predictive value (NPV) for the transient ischemic dilation values.

than 1.25 in the 2 groups of patients. Therefore, any increase in the cut-off value can exclude a significant fraction of ischemic or nonischemic patients, which makes the positive predictive value vary a lot.

Figure 1 shows a subgroup of patients with outlier or extreme values in the low probability group. This subgroup, which comprises 9 patients ( 8 women and 1 man) with transient ischemic dilation values greater than 1.30 , accounts for the limited positive predictive value of transient ischemic dilation. Extending this analysis to the subgroup of patients with a low probability of having transient ischemic dilation $>1.25$, this subgroup comprises 16 patients, 14 of whom are

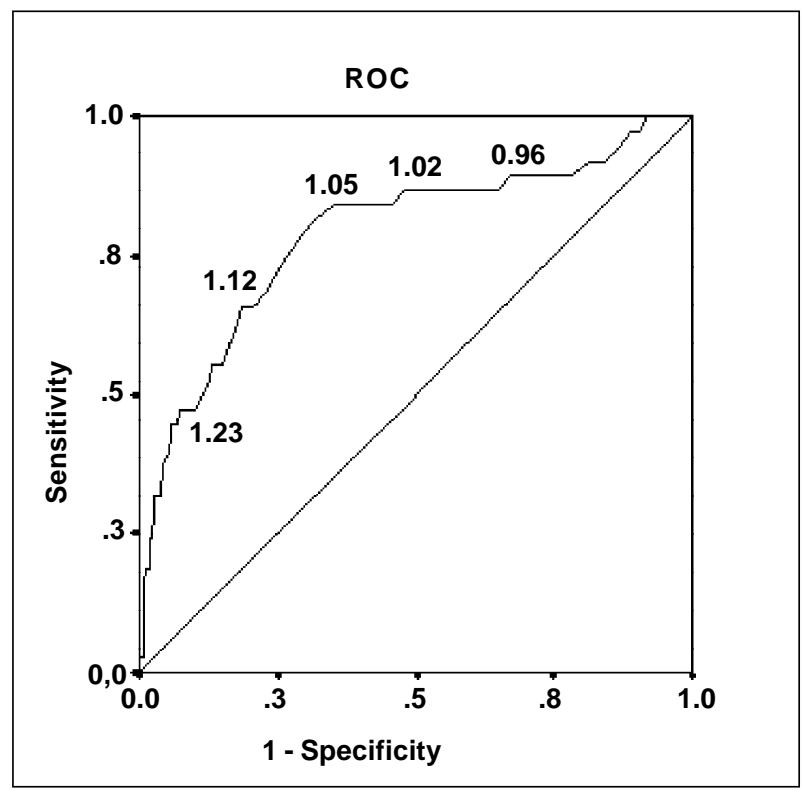

Fig. 4 - ROC curve for the transient ischemic dilation values. 
women. A possible reason for the predominance of women in this subgroup may be the difficulty of the processing software in defining the endocardial contour in patients with small ventricular cavities, mainly during the resting phase. Because the dose injected in this phase is approximately 3 times smaller than that injected in the stress phase, blurring of the ventricular wall may occur. Therefore, incorrect delimitations of the ventricular cavity occur with a tendency towards an underestimation of the ventricular volume measured at rest, with a consequent increase in transient ischemic dilation values. Thus, transient ischemic dilation assessed on myocardial perfusion scintigraphy using the 1-day protocol with stress/rest sestamibi has limitations that may be associated with the definition of the ventricular cavity at rest in patients with a small ventricle.

The low positive predictive value of transient ischemic dilation when the 1-day protocol with stress/rest sestamibi is used shows that transient ischemic dilation alone is not a good index to separate the 2 populations when used with no other information about the patient. Therefore, if we have a high transient ischemic dilation value in a patient with normal myocardial perfusion and without a high probability of disease assessed through clinical history and exercise testing, the transient ischemic dilation value should be ignored. On the other hand, in a patient with a high probability of having coronary artery disease established on exercise testing or clinical history, or both, and apparently normal myocardial perfusion study, the same transient ischemic dilation value should be valued.

In a similar study, Kinoshita et al ${ }^{4}$ reported that, for 1-day protocols using tetrofosmin stress/rest, a transient ischemic dilation value of 1.012 had sensitivity, specificity, and accuracy of $91.4 \%, 76.9 \%$, and $85 \%$, respectively. These authors studied 75 patients ( 55 with coronary artery disease and 20 healthy controls) with a high prevalence of coronary artery disease; the use of low transient ischemic dilation values in this group of patients resulted in an appropriate positive predictive value. However, in daily clinical practice, the prevalence of extensive coronary artery disease in the population examined is not very high, which may result in an inappropriate positive predictive value, if low transient ischemic dilation values are used to classify the patients as ischemic. Thus, we believe that transient ischemic dilation values greater than those used by Kinoshita et al $^{4}$ should be adopted if we want to obtain reasonable positive predictive values when using the 1-day protocol with the stress/rest sestamibi. In addition, it is worth noting that the method used for estimating the index of left ventricular dilation may vary. Kinoshita et $\mathrm{al}^{4}$ used a method other than Autoquant software, and this may have been a reason for the difference in the transient ischemic dilation values obtained for classifying the patients. With Autoquant software, a transient ischemic dilation value similar to that reported (1.01) has a sensitivity of $85 \%$ and a specificity of $53 \%$, which are lower than those obtained by those authors.

Marcassa et al ${ }^{3}$ assessed the incidence and significance of transient ventricular dilation on myocardial scintigraphy using the 2-day protocol with stress/rest sestamibi in a population of 234 consecutive men. These authors obtained a normal reference value for transient ischemic dilation (mean \pm SD) based on a group of 40 volunteers with a probability of having coronary artery disease lower than $5 \%$, and they defined abnormal dilation of the endocardium as transient ischemic dilation values above 1.24. Although the method used by Marcassa et $\mathrm{al}^{3}$ was not exactly the same as that of Autoquant software, both were based on endocardial contour. It is worth noting that taking 1.24 as a cut-off value for classifying the patients as abnormal, these authors obtained a sensitivity of $37 \%$, very similar to ours (39\%) using the same cut-off value.

In conclusion, our data showed a statistically significant difference between the transient ischemic dilation values calculated in the group of patients with reversible defects and those in the group of patients with a low probability of ischemia, when the 1-day protocol with stress/rest sestamibi was used. In clinical practice, however, this parameter should be carefully assessed, mainly in patients with a small ventricular volume, and it should only be valued in the presence of other evidence of myocardial ischemia.

\section{References}

1. Weiss AT, Berman DS, Lew AS, et al. Transient ischemic dilation of the left ventricle on stress thallium- 201 scintigraphy: a marker of severe and extensive coronary artery disease. J Am Coll Cardiol 1987; 9: 752-9.

2. Sugihara H, Katahira T, Shiga K, et al. Evaluation of transient dilation of the left ventricle on exercise thallium-201 scintigraphy. Kaku Igaku 1989; 26: 1549-53.

3. Marcassa C, Galli M, Baroffio C, Campini R, Giannuzzi P. Transient left ventricular dilation at quantitative stress-rest sestamibi tomography: clinical, electrocardiographic, and angiographic correlates. J Nucl Cardiol 1999; 6: 397-405.

4. Kinoshita N, Sugihara H, Adachi Y, et al. Assessment of transient left ventricular dilatation on rest and exercise on Tc-99m tetrofosmin myocardial SPECT. Clin Nucl Med 2002; $27:$ 34-9.

5. Mazzanti M, Germano G, Kiat H, et al. Identification of severe and extensive coronary artery disease by automatic measurement of transient ischemic dilation of the left ventricle in dual-isotope myocardial perfusion SPECT. J Am Coll Cardiol 1996; 27 : 1612-20.
6. Takeishi Y, Tono-oka I, Ikeda K, Komatani A, Tsuiki K, Yasui S. Dilatation of the left ventricular cavity on dipyridamole thallium-201 imaging: a new marker of triple-vessel disease. Am Heart J 1991; 121: 466-75.

7. Iskandrian AS, Heo J, Nguyen T, Lyons E, Paugh E. Left ventricular dilatation and pulmonary thallium uptake after single- photon emission computer tomography using thallium-201 during adenosine- induced coronary hyperemia. Am J Cardiol 1990; 66: 807-11.

8. Chouraqui P, Rodrigues EA, Berman DS, Maddahi J. Significance of dipyridamole-induced transient dilation of the left ventricle during thallium-201 scintigraphy in suspected coronary artery disease. Am J Cardiol 1990; 66: 689-94.

9. Lette J, Lapointe J, Waters D, Cerino M, Picard M, Gagnon A. Transient left ventricular cavitary dilation during dipyridamole- thallium imaging as an indicator of severe coronary artery disease. Am J Cardiol 1990; 66: 1163-70.

10. IDiretriz da Sociedade Brasileira de Cardiologia sobre Cardiologia Nuclear. Arq Bras Cardiol 2002; 78(Supl. III): 1-42. 